\title{
Association between low levels of serum miR-638 and atherosclerotic plaque vulnerability in patients with high-grade carotid stenosis
}

\author{
${ }^{*}$ Ana Luque, PhD, ${ }^{1}$ Abduljalil Farwati, PhD, Jerzy Krupinski, MD, PhD, ${ }^{2,3}$ and Josep M. Aran, $\mathrm{PhD}^{1}$ \\ ${ }^{1}$ Immune-Inflammatory Processes and Gene Therapeutics Group, Genes, Disease and Therapy Program, IDIBELL, L'Hospitalet \\ de Llobregat, Barcelona; ${ }^{2}$ Neurology Department, Mutua de Terrassa University Hospital, Terrassa, Barcelona, Spain; and \\ ${ }^{3}$ School of Healthcare Science, Manchester Metropolitan University, Manchester, United Kingdom
}

OBJECTIVE Carotid artery atherosclerosis is a major cause of ischemic stroke. However, reliable criteria to identify patients with high-risk carotid plaques beyond the severity of stenosis are still lacking. Circulating microRNAs (miRNAs) are being postulated as biomarkers for a variety of vascular immune-inflammatory diseases. The authors investigated whether cell-free circulating miR-638, highly expressed in vascular smooth muscle cells and implicated in proliferative vascular diseases, is associated with vulnerable atherosclerotic plaques in high-risk patients with advanced carotid artery stenosis undergoing carotid endarterectomy (CEA).

METHODS The authors conducted a prospective study in 22 consecutive symptomatic patients with high-grade carotid stenosis undergoing CEA and 36 age- and sex-matched patients without ischemic stroke history or carotid atherosclerosis (control group). In addition, they reviewed data from a historical group of 9 CEA patients who underwent long-term follow-up after revascularization. Total RNA was isolated from all serum samples, and relative miR-638 expression levels were detected by reverse transcription-quantitative polymerase chain reaction (RT-qPCR) and compared among groups. A correlation analysis of serum miR-638 levels with vascular risk factors and treatments, and with plaque features, was performed. The ability of serum miR-638 to discriminate between the non-CEA control group and the different CEA groups was assessed by receiver operating characteristic evaluation. A logistic regression model was employed to examine the association between stratified CEA patients and serum miR-638 levels.

RESULTS Serum levels of miR-638 were significantly lower in symptomatic CEA patients $(p=0.009)$ and particularly in the subgroup of CEA patients who had experienced stroke $(p=0.0006)$ than in non-CEA controls. Discrimination of high-risk plaques was accurate (area under the curve [AUC] 0.66 for symptomatic CEA patients in general and 0.76 for those who had experienced stroke). When only patients with high cardiovascular risk were considered, the diagnostic value of serum miR-638 from symptomatic CEA patients and CEA patients who had experienced stroke improved (AUC 0.79 and 0.85 ). Moreover, serum miR-638 was negatively correlated with the occurrence of stroke, smoker status, presence of bilateral pathology, coronary artery disease, and cholesterol treatment; and with the high-risk fibroatheroma plaques extracted from CEA patients. Multivariate logistic regression analysis demonstrated that serum miR-638 was an independent predictor of plaque instability. Furthermore, serum miR-638 appeared to attain good discrimination for atherosclerotic stenosis in CEA patients based on analysis of blood samples obtained in the historical group before and 5 years after intervention $(p=0.04)(A \cup C=0.79)$.

CONCLUSIONS According to this preliminary proof-of-concept study, serum miR-638 might constitute a promising noninvasive biomarker associated with plaque vulnerability and ischemic stroke, particularly in individuals with elevated cardiovascular risk.

https://thejns.org/doi/abs/10.3171/2018.2.JNS171899

KEYWORDS microRNA; revascularization; ischemic stroke; biomarker; atherosclerosis; vascular disorders

ABBREVIATIONS AUC = area under the curve; $\mathrm{CAD}=$ coronary artery disease $; \mathrm{CEA}=$ carotid endarterectomy $; \mathrm{CT}=$ computed tomography; miRNA = microRNA; PET $=$ positron emission tomography; ROC = receiver operating characteristic; RT-qPCR = reverse transcription-quantitative polymerase chain reaction; SCORE = Systematic Coronary Risk Evaluation; sncRNA = small noncoding RNA; VMSC = vascular smooth muscle cell.

SUBMITTED August 4, 2017. ACCEPTED February 16, 2018.

INCLUDE WHEN CITING Published online July 27, 2018; DOI: 10.3171/2018.2.JNS171899.

${ }^{*}$ J.K. and J.M.A. share senior authorship of this work. 


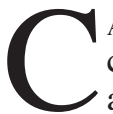
AROTID artery atherosclerosis is a major cause of ischemic stroke, which represents more than $80 \%$ of all cerebrovascular events. Several preventive measures have been developed to treat carotid artery stenosis, such as surgical plaque removal by carotid endarterectomy (CEA), endovascular stent placement, ${ }^{26,28}$ and/or pharmacological treatment. ${ }^{2}$ Nevertheless, the surgical management of asymptomatic patients with $\geq 70 \%$ carotid artery stenosis and $<10$ years of life expectancy is highly controversial due to the improvement of the medical treatment. ${ }^{13,25}$

The degree of carotid artery stenosis and plaque neovascularization, evaluated through imaging techniques such as contrast-enhanced ultrasound, positron emission tomography/computed tomography (PET/CT), and MRI, is being considered a most important predictor of carotid plaque vulnerability and, consequently, of increased risk of stroke in patients with carotid artery disease. However, most of the above advanced imaging techniques have not entered routine clinical practice because of their elevated costs. Additionally, a major limitation of these imaging techniques is their application only in advanced stages of atherosclerotic disease, because of its vessel wall anatomy/morphology-based predictive nature. ${ }^{1}$ Thus, novel diagnostic markers using minimally invasive approaches are needed for a more specific and sensitive prediction of atherosclerosis load and progression, and particularly, to reliably identify patients with high-risk carotid plaques for early and accurate stroke risk stratification.

Small noncoding RNAs (sncRNAs) are evolutionarily conserved functional epigenetic elements $(<30$ nucleotides) that have recently emerged as key posttranscriptional regulators of a variety of essential processes in living organisms at the cellular level. The major components of the sncRNA family, the microRNAs (miRNAs), generally interact specifically with the $3^{\prime}$ untranslated region of a target mRNA by sequence complementation to induce its cleavage and degradation, or a translational repression of gene expression. ${ }^{16}$ Interestingly, due to their intrinsic structural features, miRNAs are able to withstand harsh ribonuclease-rich environments such as that of plasma/ serum. ${ }^{24}$ Thus, circulating miRNAs, whether passively released by injured cells or actively delivered by intact cells, have been proposed to play a fundamental role in cell-tocell communication ${ }^{10}$ and are being postulated as useful biomarkers for a variety of conditions, such as cancer and immune-inflammatory diseases. ${ }^{9}$ Moreover, miRNAs seem to play an important role in ischemic stroke etiology and pathology, 9,10,27 and circulating miRNAs have been postulated as both biomarkers and mediators of cardiovascular diseases. . $^{71,12}$

Aberrant vascular smooth muscle cell (VSMC) proliferation and migration have been shown to play a critical role in the pathogenesis of atherosclerosis. A recent report has demonstrated that miR-638, which is highly expressed in human VSMCs, inhibits proliferation and migration of these cells by directly targeting orphan nuclear receptor NOR1, a critical regulator implicated in proliferative vascular diseases. ${ }^{20}$ Moreover, it has been shown that miR638 may be secreted into extracellular spaces through sphingomyelin phosphodiesterase $3 .{ }^{19}$ Thus, our aim was to explore the potential of cell-free circulating miR-638 as an atherosclerosis-related, early predictive, and minimally invasive biomarker of carotid plaque vulnerability and ischemic stroke risk in patients with high-grade carotid artery stenosis. We took into account its relevance in stroke pathophysiology, revealed by bioinformatics and in vitro cell culture analyses. The methodology and results of these analyses are presented in the Supplemental Material (accessible online).

\section{Methods \\ Subjects and Clinical Evaluation}

All subjects in this report were selected from a cohort of consecutive inpatients with different degrees of stenosis, recruited at the Mutua de Terrassa University Hospital from 2013 to 2015 . The study was approved by the IDIBELL's ethics committee in accordance with institutional guidelines and the Declaration of Helsinki, and the patients' written informed consent was obtained. We prospectively collected serum samples from 22 patients of Western European descent (age range 42-81 years) who underwent CEA for symptomatic stenosis. The samples were collected on the day of surgery. All patients had experienced transient ischemic attacks, amaurosis fugax, or ipsilateral stroke. The patients had a high degree of carotid artery stenosis ( $\geq 60 \%$ on echo Doppler imaging confirmed by MR angiography or CT angiography, according to NASCET criteria). All 22 patients underwent CEA within 3 weeks after the symptomatic event. According to morphological and histological criteria, CEA-extracted plaques had instability features such as inflammatory activity, thin fibrous cap, large lipid core, visible ulceration, intraplaque hemorrhage, and rupture, corresponding to lesion type $\mathrm{V}$ or VI of the classification of the American Heart Association. ${ }^{32}$ Thirty-six age- and sex-matched patients (age range 43-84 years) constituted a control group. Thirty of the controls had presented to the neurology unit of the same institution with complaints of headache, migraine, vertigo, or dizziness and had no history of stroke or carotid atherosclerosis (all had $<30 \%$ stenosis on carotid echo Doppler imaging), and 6 of the controls were patients with an old history of healed ( $>5$ years recovered) hemorrhagic stroke. All non-CEA patient controls underwent blood extraction at hospital discharge. Patients with a known history of acute arterial or venous thromboembolism, active infections, renal failure, hepatic disease, neoplasms, recent trauma, or surgery were excluded from the study.

Standard hematological and biochemical analyses were routinely performed at the hospital laboratory. Patients' basic clinical data and vascular risk factors, including previous antihypertensive, statin, and antiplatelet treatment history, are summarized in Table 1.

In an independent pilot analysis, we analyzed data obtained in a historical group of 9 additional CEA patients (age range 57-83 years) before and 5 years after surgical intervention. The clinical characteristics of these patients are shown in Supplementary Table 1.

The cardiovascular risk for both the CEA group and the control group was estimated according to the Systematic Coronary Risk Evaluation (SCORE) prediction model calibrated for use in Spain. ${ }^{29}$ 
TABLE 1. Main clinical characteristics of the symptomatic CEA patients

\begin{tabular}{|c|c|c|c|c|c|}
\hline \multirow[b]{2}{*}{ Characteristic } & \multicolumn{2}{|c|}{$\begin{array}{c}\text { Symptomatic } \\
\text { CEA Pts }\end{array}$} & \multicolumn{2}{|r|}{ Controls } & \multirow{2}{*}{$\begin{array}{c}\mathrm{p} \\
\text { Value }\end{array}$} \\
\hline & $n$ & Value & $n$ & Value & \\
\hline Age (yrs), mean & 22 & $63.8 \pm 11.0$ & 36 & $67.7 \pm 13.4$ & 0.25 \\
\hline Sex: male & 22 & $17(77.3)$ & 36 & $21(58.3)$ & 0.12 \\
\hline Smoking & 22 & $10(45.5)$ & 36 & $10(27.8)$ & 0.14 \\
\hline Dyslipidemia & 22 & $14(63.6)$ & 36 & $16(44.4)$ & 0.13 \\
\hline HTN & 22 & $19(86.4)$ & 36 & $21(58.3)$ & 0.02 \\
\hline Diabetes & 22 & $7(31.8)$ & 36 & $8(22.2)$ & 0.31 \\
\hline PVD & 22 & $5(22.7)$ & 36 & $5(13.9)$ & 0.30 \\
\hline CAD & 22 & $3(13.6)$ & 36 & $1(2.8)$ & 0.15 \\
\hline Cerebrovascular event & 22 & $22(100.0)$ & 36 & $0(0.0)$ & 0.00 \\
\hline Bilat pathology $>50^{*}$ & 20 & $8(40.0)$ & 36 & $6.0(16.7)$ & 0.06 \\
\hline Fibrinogen $(\mathrm{g} / \mathrm{L})$, mean & 22 & $5.1 \pm 1.5$ & 36 & $4.3 \pm 1.2$ & 0.03 \\
\hline $\begin{array}{l}\text { Total cholesterol } \\
\text { (mmol/L), mean }\end{array}$ & 22 & $4.5 \pm 1.2$ & 36 & $3.7 \pm 1.2$ & 0.02 \\
\hline LDL-C (mmol/L), mean & 22 & $2.4 \pm 1.1$ & 36 & $2.2 \pm 0.7$ & 0.30 \\
\hline $\mathrm{HDL}-\mathrm{C}(\mathrm{mmol} / \mathrm{L})$, mean & 22 & $1.3 \pm 0.5$ & 36 & $1.2 \pm 0.3$ & 0.81 \\
\hline TG (mmol/L), mean & 22 & $1.4 \pm 0.5$ & 36 & $1.9 \pm 1.0$ & 0.05 \\
\hline ESR (mm/hr), mean & 21 & $15.1 \pm 10.8$ & 35 & $14.7 \pm 10.9$ & 0.89 \\
\hline WBC $\times 10^{6} / \mathrm{L}$, mean & 22 & $7065 \pm 2407$ & 30 & $7744 \pm 2597$ & 0.33 \\
\hline $\begin{array}{l}\text { Creatinine }(\mathrm{mg} / \mathrm{dl}) \text {, } \\
\text { mean }\end{array}$ & 22 & $0.8 \pm 0.2$ & 34 & $1.0 \pm 1.0$ & 0.52 \\
\hline SBP (mm Hg), mean & 22 & $156.0 \pm 32.3$ & 36 & $139.2 \pm 36.8$ & 0.09 \\
\hline Antiplatelet treatment & 20 & $19(95.0)$ & 36 & $11(30.6)$ & 0.00 \\
\hline SBP treatment & 22 & $19(86.4)$ & 36 & $18(50.0)$ & 0.01 \\
\hline Cholesterol treatment & 21 & $15(71.4)$ & 36 & $14(38.9)$ & 0.02 \\
\hline
\end{tabular}

$\mathrm{CAD}=$ coronary artery disease; $\mathrm{ESR}=$ erythrocyte sedimentation rate; $\mathrm{HDL}-\mathrm{C}$ = high-density lipoprotein cholesterol; HTN = hypertension; LDL-C = low-density lipoprotein cholesterol; Pts = patients; PVD = peripheral vascular disease; $\mathrm{SBP}=$ systolic blood pressure; $\mathrm{TG}=$ triglycerides; $\mathrm{WBC}=$ white blood cells . Data are reported as $n(\%)$ unless otherwise indicated; mean values are reported \pm SD. Boldface type indicates statistical significance $(p<0.05)$.

* More than $50 \%$ contralateral stenosis on ultrasound.

\section{Blood Sample Processing and RNA Extraction}

The serum fractions from CEA and control patients were obtained according to standard protocols. Briefly, blood samples were collected in EDTA-containing tubes immediately prior to the CEA procedure and, selectively in the 9 additional patients included in the pilot analysis, 5 years after CEA. All blood samples were processed within 1 hour by centrifugation at $2000 \mathrm{~g}$ for 10 minutes at $4^{\circ} \mathrm{C}$, and the supernatants were quickly removed, aliquoted, and stored immediately at $-80^{\circ} \mathrm{C}$. For analysis, serum samples were thawed on ice and centrifuged at $3000 \mathrm{~g}$ for $5 \mathrm{~min}$ utes, avoiding the presence of traces of red blood cells and other cellular debris that might affect the miRNA analysis.

RNA was isolated from $200 \mu \mathrm{l}$ of serum using the miRNeasy Mini Kit (Qiagen). Variability in sample RNA isolation was taken into account by normalization. This procedure included addition of the synthetic Caenorhabditis elegans miRNA cel-miR-54 (5 fmol) (Integrated DNA Technologies) to each denatured serum sample (af- ter the initial RNA extraction step) to avoid its degradation by endogenous serum RNases.

\section{Real-Time RT-PCR Quantification}

A fixed volume of $5 \mu \mathrm{l}$ of RNA per individual sample was reverse transcribed using the TaqMan microRNA reverse transcription (RT) kit (Applied BioSystems) and 2 $\mu l$ of miR-638-specific stem-loop primers from the TaqMan miRNA assay (hsa-miR-638, ID 001582; mature miRNA sequence: 5'-AGGGAUCGCGGGCGGGUGG CGGCCU- ${ }^{\prime}$ ) in a small-scale RT reaction (comprising $1.10 \mu \mathrm{l}$ of nuclease-free water, $1 \mu \mathrm{l}$ of $10 \times \mathrm{RT}$ buffer, $0.13 \mu \mathrm{l}$ of RNase-inhibitor [20 U/ml], $0.10 \mu \mathrm{l}$ of $100 \mathrm{mM}$ dNTPs, and $0.67 \mu \mathrm{l}$ of Multiscribe reverse transcriptase [50 U/ml]), using a GeneAmp 9700 PCR thermocycler system (Applied BioSystems) according to the manufacturer's instructions. To enhance sensitivity, a 2.5- $\mu$ l aliquot of undiluted RT product was combined with 7.5 $\mu l$ of preamplification solution $(5 \mu$ of TaqMan PreAmp Master Mix and $2.5 \mu$ of $0.2 \times$ TaqMan miRNA Assay) and amplified in the GeneAmp 9700 PCR thermocycler system (Applied BioSystems) by heating to $95^{\circ} \mathrm{C}$ for 10 minutes, followed by 10 cycles of $95^{\circ} \mathrm{C}$ for 15 seconds and $60^{\circ} \mathrm{C}$ for 4 minutes.

Finally, $2.5 \mu \mathrm{l}$ of water-diluted (1:5) preamplified product was combined with $5 \mu \mathrm{l}$ of TaqMan Universal PCR Master Mix with no AmpErase UNG (Applied BioSystems), $0.5 \mu$ l of $20 \times$ TaqMan microRNA assay, and $2 \mu \mathrm{l}$ of nuclease-free water in a 10- $\mu$ l polymerase chain reaction (PCR). Real-time PCR was carried out on an Applied BioSystems $7900 \mathrm{HT}$ thermocycler $\left(50^{\circ} \mathrm{C}\right.$ for 2 minutes and $95^{\circ} \mathrm{C}$ for 10 minutes, followed by 40 cycles of $95^{\circ} \mathrm{C}$ for 15 seconds and $60^{\circ} \mathrm{C}$ for 1 minute). Data were analyzed with SDS 2.4 (Applied BioSystems). The amplification curves were individually inspected and miRNAs with abnormal amplification patterns were removed from analysis. The spiked-in cel-miR-54 was measured in parallel to the miRNA-638 (cel-miR-54 ID 001361). All reactions were run in triplicate. For each sample, the relative abundance of target miRNA-638 was determined by the equation $2^{-\Delta \mathrm{Ct}}$, in which $\Delta \mathrm{Ct}=\mathrm{Ct}_{\text {miR-638 }}-\mathrm{Ct}_{\text {cel-miR-54 }}$, and transformed using the natural logarithm.

\section{Statistical Analysis}

Clinical-pathological parameters of CEA and control non-CEA groups were described using means and standard deviations for continuous variables and frequencies for categorical variables and compared using the t-test for independent samples or the chi-square test, respectively. Normal distribution of serum miR-638 levels was assessed by quantile-quantile plot and Shapiro-Wilks test. The t-test or the Wilcoxon-Mann-Whitney test, depending on the normality of the distribution, was applied to assess significant differences between CEA and control groups or within a historical CEA group at 0 and 5 years postintervention. The relationship of miRNA-638 with vascular risk factors and treatments, and with plaque features, was analyzed by Spearman's rank correlation or by point-biserial correlation, where appropriate. The ability of serum miR638 levels to discriminate between the non-CEA control group and the CEA patient group, the non-CEA control 
group and the CEA stroke subgroup, or a historical CEA group at 0 and 5 years postintervention, was assessed using receiver operating characteristic (ROC) analysis. To analyze the association between high-risk atherosclerotic plaque (CEA) and serum miR-638 levels a logistic regression model was estimated. CEA/non-CEA control was used as the dependent variable and a raw and adjusted odds ratio for 1 unit change of miR-638 was calculated. Adjusting variables included classical vascular risk factors (hypertension, coronary artery disease [CAD], fibrinogen, and cholesterol) with a statistically significant association between CEA and non-CEA controls (Table 1). All tests were 2 -tailed, and $p<0.05$ was considered statistically significant. Statistical analyses were conducted using $\mathrm{R}$ version 3.2.2 for Windows, SPSS software version 15.0 (SPSS Inc.) and GraphPad Prism version 6 (GraphPad Software Inc.).

\section{Results}

\section{Characteristics of the Study Population}

This study was carried out in 2 well-defined populations: patients at very high risk for atherothrombotic events and individuals at low risk for atherothrombotic events. A total of 22 patients undergoing CEA for high-grade stenosis, including 11 patients with ischemic stroke, 8 patients who had TIAs, and 3 patients experiencing amaurosis (CEA group), and 36 age- and sex-matched individuals visiting the neurology unit for conditions unrelated to atherosclerotic disease (control non-CEA group) were incorporated in the study. The CEA patients and non-CEA controls displayed similar characteristics except for a significantly higher incidence of hypertension and significantly increased fibrinogen and total cholesterol values in the CEA patients. Moreover, the numbers of patients receiving antiplatelet, cholesterol-lowering, and/or blood pressure-lowering treatments were also significantly higher in the CEA group (Table 1). In the symptomatic CEA patient group, those having experienced ischemic stroke $(\mathrm{n}=11)$ were all male and showed a significantly greater prevalence of smoking, increased levels of total cholesterol, and increased systolic blood pressure compared to the nonCEA control group (Supplementary Table 2).

\section{Decreased Circulating miR-638 in CEA Patients}

miR-638 seems to constitute a relevant vascular miRNA participating in stroke pathophysiology and, therefore, a potential biomarker of carotid artery disease (see Supplemental Information and Tables).

In the population of symptomatic patients with carotid stenosis undergoing CEA, the serum level of miR-638 was significantly lower than in individuals without atherosclerosis (non-CEA control) $(\mathrm{p}=0.009)$ (Fig. 1A). The ability of serum miR-638 to differentiate the CEA group from the non-CEA control group was estimated by the ROC curve, with an area under the curve (AUC) of 0.66 (95\% CI 0.52 $0.81, \mathrm{p}=0.04$ ) (Fig. 1B). Furthermore, the difference between the serum levels of miR-638 from the stroke patient subgroup undergoing CEA and those from the non-CEA control group reached the highest statistical significance $(\mathrm{p}=0.0006)$ (Fig. 1C). Consequently, the discriminatory capability of serum miR-638 from the stroke CEA patient
TABLE 2. Correlation of serum miR-638 levels with clinical parameters and vascular risk factors in the study population

\begin{tabular}{|c|c|c|c|}
\hline Correlation \& Parameter & $\rho$ & $r_{b s}$ & $\mathrm{p}$ Value \\
\hline \multicolumn{4}{|l|}{ Spearman's rank correlation } \\
\hline Age (yrs) & 0.017 & & 0.897 \\
\hline Fibrinogen (g/L) & 0.017 & & 0.901 \\
\hline Total cholesterol (mmol/L) & 0.023 & & 0.863 \\
\hline LDL-C (mmol/L) & 0.083 & & 0.537 \\
\hline HDL-C (mmol/L) & -0.252 & & 0.056 \\
\hline $\mathrm{TG}(\mathrm{mmol} / \mathrm{L})$ & 0.469 & & 0.000 \\
\hline $\mathrm{ESR}(\mathrm{mm} / \mathrm{hr})$ & 0.074 & & 0.581 \\
\hline $\mathrm{WBC} \times 10^{6} / \mathrm{L}$ & 0.071 & & 0.595 \\
\hline Creatinine (mg/dl) & -0.008 & & 0.952 \\
\hline $\mathrm{SBP}(\mathrm{mg} / \mathrm{dl})$ & 0.052 & & 0.697 \\
\hline \multicolumn{4}{|l|}{ Biserial correlation } \\
\hline Sex & & 0.152 & 0.254 \\
\hline Smoking & & -0.263 & 0.046 \\
\hline Dyslipemia & & -0.153 & 0.250 \\
\hline HTN & & -0.104 & 0.439 \\
\hline Diabetes & & 0.151 & 0.257 \\
\hline PVD & & -0.009 & 0.945 \\
\hline CAD & & -0.323 & 0.013 \\
\hline Ischemic stroke & & -0.339 & 0.009 \\
\hline Bilat pathology $>50^{*}$ & & -0.345 & 0.009 \\
\hline Antiplatelet treatment & & -0.258 & 0.055 \\
\hline SBP treatment & & -0.232 & 0.079 \\
\hline Cholesterol treatment & & -0.372 & 0.004 \\
\hline
\end{tabular}

$\rho=$ Spearman's correlation coefficient; $r_{b s}=$ biserial correlation coefficient. Boldface type indicates statistical significance $(p<0.05)$.

${ }^{*}$ More than $50 \%$ contralateral stenosis on ultrasound.

subgroup increased compared to that of serum miR-638 from the non-CEA control group (AUC $=0.76,95 \% \mathrm{CI}$ $0.59-0.96, p=0.01)$ (Fig. 1D).

Furthermore, in our whole cohort (CEA patients and controls), serum miR-638 was negatively correlated with the occurrence of stroke, smoker status, presence of bilateral pathology, CAD, and cholesterol treatment (Table 2). Conversely, serum miR-638 levels correlated positively with blood triglyceride levels. Several studies have shown that poor outcomes after acute ischemic stroke were closely related to lower serum triglyceride levels, not hypertriglyceridemia. ${ }^{5}$

After logistic regression, the level of serum miR-638 was associated with the presence of vulnerable plaques and therefore with stroke risk in both the CEA symptomatic patient group and the stroke subgroup (Table 3).

Finally, in the CEA patients, serum levels of miR-638 were correlated with major features of the high-grade stenotic CEA plaques (Table 4). A strong negative correlation was found for high-risk fibroatheroma plaques. Conversely, a strong positive correlation was found with culprit plaques (particularly ruptured and ulcerated/hemorrhagic plaques). These results highlight the potential predictive utility of serum miR-638 for vulnerable plaques, remarkably those that have not yet progressed to an overt open lesion. 


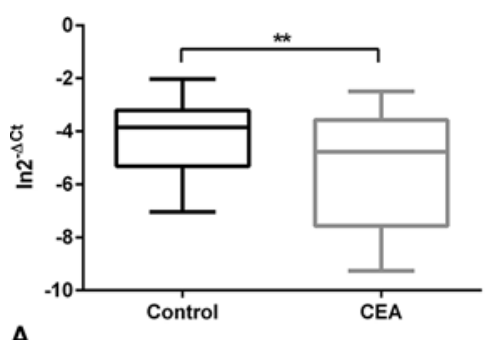

A
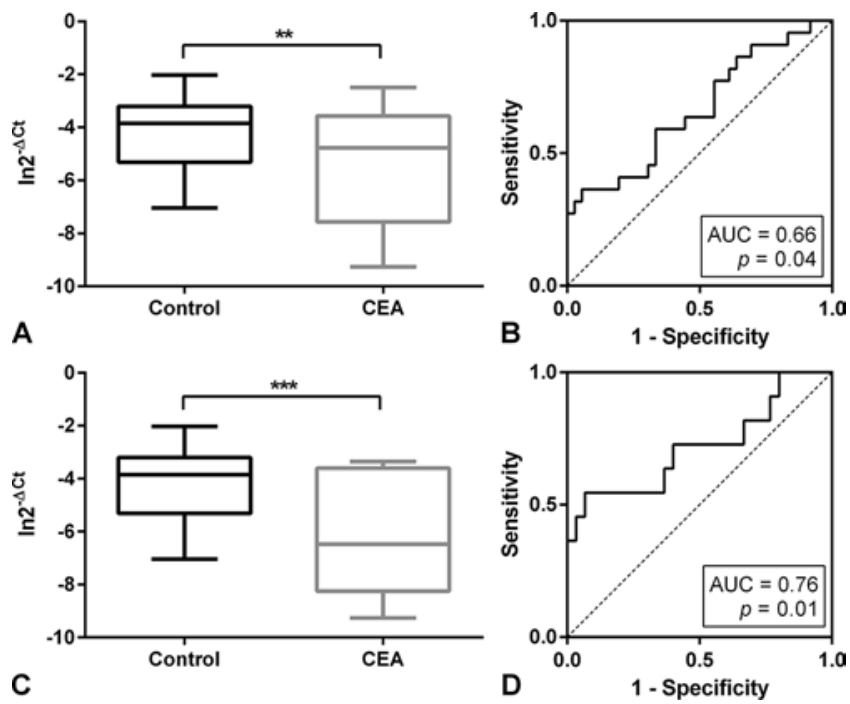

FIG. 1. Serum levels and diagnostic accuracy of miR-638 in the symptomatic CEA patient group and stroke subgroup compared to the nonCEA control group. A and C: Serum levels of miR-638 assessed by reverse transcription-quantitative polymerase chain reaction (RT-qPCR) are reduced in symptomatic CEA patients $(n=22)(A)$ and stroke CEA patients $(n=11)(C)$ compared to controls $(n=36)\left({ }^{* *} p<0.01\right.$; ${ }^{* * *} p<$ 0.001 ). In $2^{-\Delta c t}, \mathrm{miR}-638$ levels relative to cel-miR-54, transformed into linear form using the formula $2^{-\Delta C t}$. All quantities have been transformed using the natural logarithm. The thick black line inside the boxes indicates the median. The top and bottom of the boxes indicate 75th and 25th percentiles, respectively. B and D: ROC analysis was used to determine the diagnostic accuracy of serum miR-638 predicting CEA intervention for high-risk ischemic stroke. Discrimination of symptomatic CEA patients from controls (B), AUC 0.66 (95\% Cl 0.52-0.81). Discrimination of stroke CEA patients from controls (D), AUC $0.76(95 \% \mathrm{Cl}$ $0.59-0.96$ ). The dashed lines indicate the reference line (AUC $=0.5$ ).

\section{Effect of SCORE on Accuracy of Circulating miR-638 for Vulnerable Carotid Plaque Prediction}

Since CAD was significantly correlated with decreased serum miR-638 levels, we further evaluated whether focusing on the analysis of serum miR-638 to high-cardiovascular risk individuals using the SCORE prediction model $^{29}$ (SCORE $>5 ; 8$ patients from the symptomatic CEA group and 14 from the non-CEA control group; Supplementary Table 5) would improve its diagnostic accuracy. We confirmed that the serum levels of miR-638 were significantly lower in the symptomatic CEA + SCORE $>5$ subgroup $(\mathrm{n}=8)$ (Fig. 2A) and the stroke CEA + SCORE $>5$ subgroup $(\mathrm{n}=7)$ (Fig. $2 \mathrm{C})$, compared to the non-CEA control + SCORE $>5$ subgroup $(n=14)(p=0.009$ and $p$ $=0.003$, respectively). We also stratified the ROC analysis of serum miR-638 levels to assess the sensitivity and specificity of vulnerable plaque and stroke risk prediction among the above-described subgroups with SCORE $>5$ and obtained an improved diagnostic accuracy (symptomatic CEA + SCORE $>5$ subgroup vs non-CEA control + SCORE $>5$ subgroup, AUC $=0.79,95 \%$ CI $0.59-1.00, p$ $=0.02$ [Fig. 2B]; stroke CEA + SCORE $>5$ subgroup vs non-CEA control + SCORE $>5$ subgroup, AUC $=0.85$, 95\% CI 0.66-1.04, $\mathrm{p}=0.01$ [Fig. 2D]).

Multivariate logistic regression analysis demonstrated that the serum miR-638 level was also associated with carotid plaque instability in high-cardiovascular risk individuals (adjusted $\mathrm{OR}=0.51,95 \%$ CI $0.24-0.87, \mathrm{p}=0.032$ ) (Table 3).

\section{Prognostic Value of Circulating miR-638 in Long-Term Follow-Up CEA Patients}

We further assessed whether serum miR-638 might be useful to predict the outcome of aggressive medical therapy (CEA plus pharmacological treatment with risk factor modification) on atherosclerotic disease progression in the long term. We analyzed serum miR-638 levels in blood samples obtained from 9 CEA patients just before intervention and 5 years after CEA. Smoking status proved the only risk factor for which there was a significant difference between the 2 time points (Supplementary Table 1), and this was presumably because medical management of CEA patients advises smoking cessation. The mean serum levels of miR-638 were significantly greater at 5 years after CEA ( $p=0.04$ ) (Fig. 3, left). Moreover, serum miR-638 showed good discrimination for atherosclerotic carotid stenosis in CEA patients before and after long-term postintervention follow-up (AUC $=0.79,95 \%$ CI $0.57-1.01, \mathrm{p}$ $=0.04$ ) (Fig. 3, right).

Of note, 5 years after CEA the cohort of 9 CEA patients could be classified into 2 subgroups, with slightly significant divergences $(p=0.045)$ regarding the dynamics of miR-638 in serum: 1) a group of 4 individuals with miR638 levels remaining stable from 0 to 5 years post-CEA and 2) a group of 5 individuals displaying an increase in serum miR-638 levels from 0 to 5 years post-CEA (Fig. 3, left). No statistically significant differences could be established between the above groups regarding the risk factors and biochemical parameters analyzed. Nevertheless, the 2 unique CEA patients affected by peripheral vascular disease in this cohort had the lowest serum miR-638 expression levels up to 5 years post-CEA (Fig. 3, left).

\section{Discussion}

In this preliminary report, we describe for the first time the association of serum miR-638 with atherosclerotic carotid artery disease, comparing 2 well-defined groups of patients at different risk for atherothrombotic events. Thus,

TABLE 3. Raw and adjusted odds ratios for the association between serum miR-638 and CEA by multivariate logistic regression model

\begin{tabular}{lcccccc}
\hline \multicolumn{1}{c}{ Variable } & OR & $95 \% \mathrm{Cl}$ & $\mathrm{p}$ Value & Adjusted OR* & $95 \% \mathrm{Cl}$ & $\mathrm{p} \mathrm{Value}$ \\
\hline Symptomatic pts & 0.65 & $0.45-0.90$ & 0.015 & 0.63 & $0.42-0.90$ & 0.016 \\
\hline Stroke pts & 0.50 & $0.29-0.77$ & 0.004 & 0.44 & $0.23-0.71$ & 0.003 \\
\hline Pts w/ SCORE $>5$ & 0.51 & $0.26-0.84$ & 0.020 & 0.51 & $0.24-0.87$ & 0.032 \\
\hline
\end{tabular}

${ }^{*} \mathrm{CAD}$ and fibrinogen not used in the model due to estimation problems in standard errors. 
TABLE 4. Correlation of serum miR-638 levels with instability features of the atherosclerotic plaques from CEA patients

\begin{tabular}{lcccccc}
\hline & \multicolumn{4}{c}{ Biserial Correlation } \\
\cline { 2 - 3 } \multicolumn{1}{c}{ Feature } & \multicolumn{2}{c}{ Symptomatic CEA Pts } & & \multicolumn{2}{c}{ Stroke CEA Pts } \\
\cline { 2 - 3 } \cline { 5 - 6 } & $r_{b s}$ & $p$ Value & & $r_{b s}$ & $p$ Value \\
\hline Ruptured plaque & 0.839 & $\mathbf{0 . 0 0 0}$ & & 0.835 & 0.001 \\
\hline Ulcerated plaque & 0.443 & 0.044 & & 0.598 & 0.052 \\
\hline Fibroatheroma & -0.708 & 0.000 & & -0.692 & 0.018 \\
\hline
\end{tabular}

Boldface type indicates statistical significance $(p<0.05)$.

using the CEA procedure as a surrogate endpoint, we have shown that serum miR-638 levels are inversely correlated with carotid artery atherosclerotic disease and high-grade stenosis leading to ischemic cerebrovascular events.

Although the concise role of miR-638 in vascular pathophysiology is largely unknown, it has been shown that this particular miRNA inhibits PDGF-BB-induced human VSMC proliferation and migration by targeting the NOR1/ cyclin D pathway. ${ }^{20}$ Conversely, most atherothrombotic events are caused by rupture of a vulnerable plaque with a thin VSMC-poor fibrous cap overlying a relatively large necrotic core. Thus, VSMC apoptosis reduces fibrous cap thickness, increases necrotic cores, increases plaque inflammation (all processes predicted to promote plaque instability), ${ }^{3}$ and could account for the reduced levels of circulating miR-638 that we observed in patients with high-grade carotid stenosis. Another recent study has demonstrated extracellular vesicle-mediated transfer of the atheroprotective miRNAs miR-143/145 between endothelial cells and VSMCs, providing compelling evidence that intercellular transport of miRNAs can influence atherosclerosis. ${ }^{14}$ Downregulation of miR-638 has also been associated with hypertension. ${ }^{23}$ Furthermore, miR-638 might regulate gene expression pathways related to the oxidative stress response and aging, ${ }^{6}$ of relevance in the atherogenesis process. On the other hand, we have shown the presence of miR-638 in endothelial cells and its downregulation by pro-inflammatory stimuli. In addition, our bio-informatics analyses uncovered potential miR-638 targets, such as the transcription factor MAFB, a novel regulator of human adipose tissue inflammation, whose polymorphisms have been associated with increased risk of CAD and ischemic stroke, ${ }^{35}$ and signaling pathways putatively involved in stroke, such as NF- $\kappa \mathrm{B}$, nitric oxide, SODD/TNFR1, and eicosanoid metabolism. All together, the above hints predict a relevant role for miR-638 in stroke pathophysiology and support the analysis of circulating miR-638 as a noninvasive biomarker for carotid artery disease leading to stroke.

We found reduced serum miR-638 levels in symptomatic high-grade stenosis patients undergoing CEA. Accordingly, serum miR-638 levels inversely correlated with several vascular risk factors, including occurrence of stroke, CAD, smoking status, and presence of bilateral pathology ( $>50 \%$ contralateral stenosis), and with cholesterol treatment. Ischemic stroke and CAD share several risk factors and many aspects of their underlying pathophysiology. ${ }^{17}$ Thus, our study further indicates that serum miR-638 levels might constitute a useful biomarker for vulnerable atherothrombotic plaque identification, ische-
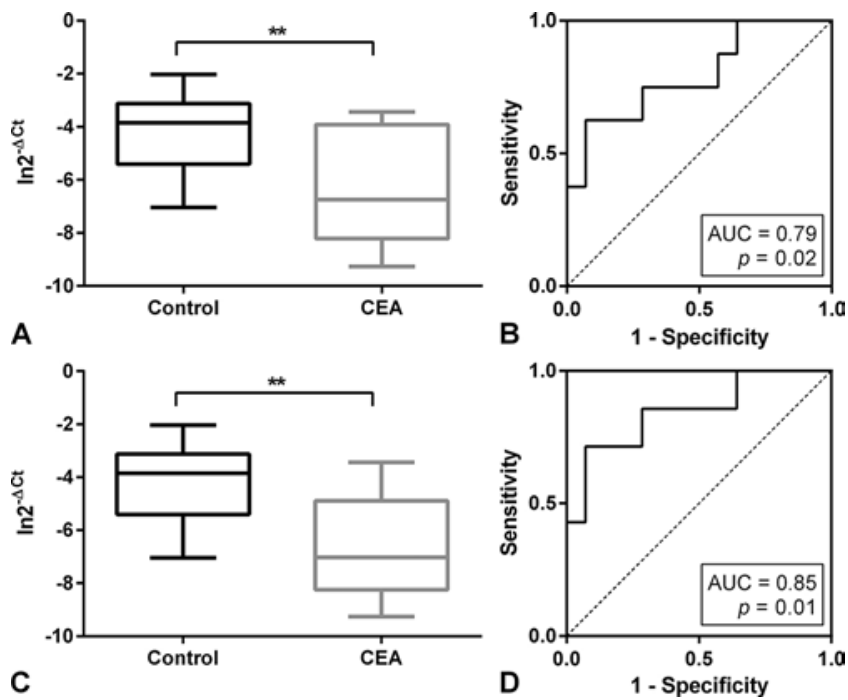

FIG. 2. Serum levels and diagnostic accuracy of miR-638 individuals with high cardiovascular risk (SCORE > 5). A and C: Serum levels of miR-638 assessed by RT-qPCR are reduced in symptomatic CEA patients with SCORE > $5(n=8)(A)$ and stroke CEA patients with SCORE $>5(n=7)(C)$ compared to control non-CEA individuals with SCORE $>5(n=14)\left({ }^{* *} p<0.01\right)$. In $2^{-\Delta C t}$, miR-638 levels relative to cel-miR-54, transformed into linear form using the formula $2^{-\Delta C t}$. All quantities have been transformed using the natural logarithm. The thick black line inside the boxes indicates the median. The top and bottom of the boxes indicate 75th and 25th percentiles, respectively. B and D: ROC analysis was used to determine the diagnostic accuracy of serum miR-638 predicting CEA intervention for high-risk ischemic stroke in high-cardiovascular risk individuals (SCORE > 5) with the highest sensitivity and specificity. Discrimination of symptomatic CEA patients from non-CEA controls (B), AUC $0.79(95 \% \mathrm{Cl} 0.59-1.00)$. Discrimination of stroke CEA patients from non-CEA controls (D), AUC $0.85(95 \% \mathrm{Cl} 0.66-1.04)$. The dashed lines indicate the reference line (AUC $=0.5)$.

mic stroke risk prediction, and selection of patients for vascular intervention among individuals with high-grade stenosis and high-risk cardiovascular disease, achieving its highest diagnostic accuracy in this cohort.

Serum miR-638 determination might also be useful for long-term management, monitoring, and/or outcome prediction after carotid revascularization. The small historical group of patients who underwent CEA and had 5 years of post-CEA follow-up experienced no significant variation in the classical vascular risk factors analyzed except for smoking cessation. It has been reported that smoking cessation alone decreases stroke risk substantially over a 5-year period in comparison to the risk for continuing smokers. ${ }^{18,34}$ Thus, serum miR-638 determination appeared to discriminate pre-CEA and post-CEA in these patients. Importantly, the concomitant presence of peripheral vascular disease in the CEA patients suggested poor prognosis, based on the low serum miR-638 levels found in the 2 patients with peripheral vascular disease at 5 years of follow-up. It has been reported that progressive peripheral vascular disease is associated with increased stroke risk, ${ }^{8}$ and the prevalence of symptomatic carotid stenosis was associated with a history of peripheral vascular disease. ${ }^{22}$

So far, no serum biomarker has qualified for standard clinical use in carotid artery atherosclerotic disease. Nevertheless, promising protein-based candidates involved in 

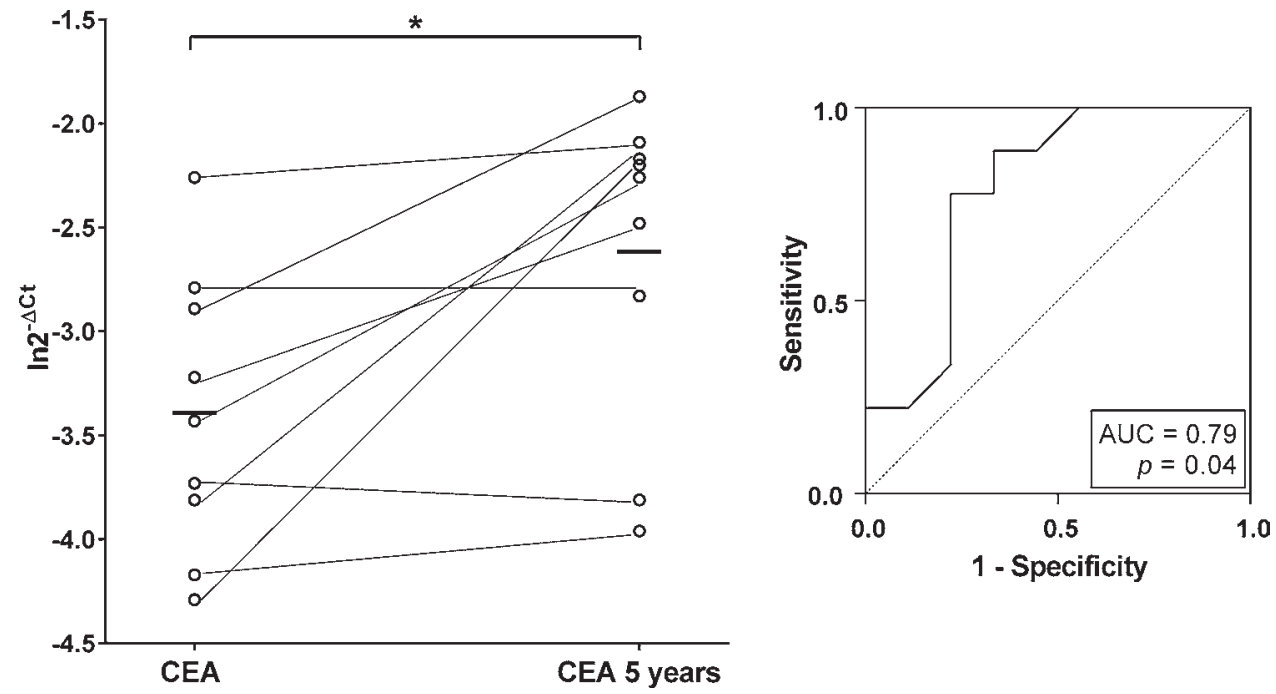

FIG. 3. Prognostic accuracy of serum miR-638 in a historical group of CEA patients $(n=9)$ with long-term follow-up. Left: Comparison of serum miR-638 levels obtained before and 5 years after CEA ( $\left.{ }^{*} p<0.05\right)$. Right: ROC analysis to assess the discrimination potential of serum miR-638 levels for CEA before and at 5 years after revascularization, AUC $0.79(95 \% \mathrm{Cl} 0.57-1.01)$.

inflammation, lipid accumulation, apoptosis, proteolysis, thrombosis, and angiogenesis are often proposed to uncover plaque vulnerability and stroke risk..$^{15}$ Circulating miR-638 determination may be less technically demanding, more cost-effective, and therefore more feasible for wider, highthroughput routine screening of patients with carotid artery disease than duplex ultrasound. ${ }^{4}$ The joint determination of miR-638 either with classical vascular risk factors or with other recently described and potential serum biomarkers, such as other miRNAs, ${ }^{21,33}$ proteins,${ }^{31}$ or cell-derived microparticles, ${ }^{30}$ may increase even further the reliability and precision of ischemic stroke prediction and prognosis.

\section{Study Limitations}

The low number of patients recruited in this preliminary proof-of-concept study, although clinically well characterized, might hamper its relevance despite the robustness of the statistical analyses performed. Therefore, future largescale prospective studies are needed to confirm circulating miR-638 for the prediction of ischemic stroke in patients with high-grade stenosis and to establish whether circulating miR-638 levels display prognostic value with respect to the occurrence of neurological events. It might be also worthwhile to explore the diagnostic and prognostic potential of circulating miR-638 in high-risk individuals who have a less severe degree of stenosis (50\%-60\%), a population that was not represented in our study. Finally, although several hypotheses have been formulated, our study did not cover the analysis of the molecular mechanism leading to miR-638 reduction in the serum from CEA patients and the real involvement of miR-638 in the atherogenic process.

\section{Conclusions}

We suggest cell-free circulating miR-638 as a novel and independent biomarker for vulnerable atherothrombotic plaque identification in patients with high-grade carotid artery stenosis and, consequently, for primary and second- ary ischemic stroke risk prediction, particularly in highcardiovascular risk individuals. Moreover, circulating miR-638 might also be a potential prognostic biomarker for monitoring the effectiveness of medical treatment, and perhaps for the occurrence of restenosis.

\section{Acknowledgments}

We thank Cristian Tebé, from the IDIBELL's Statistical Consultancy Service, for his help with the statistical analyses.

Josep M. Aran received support from the Ministerio de Economía y Competitividad (Madrid, Spain) (grant FIS-ISCIII PI10/1073, co-funded by FEDER funds/European Regional Development Fund [ERDF]-a way to build Europe) and from the Generalitat de Catalunya (grant 2009SGR1490). Jerzy Krupinski received support from FMT grant-2012 (Terrassa, Fundació per Docencia i Recerca, Mútua Terrassa). Abduljalil Farwati was supported by fellowship 2010FI-B 00299 (Generalitat de Catalunya). Josep M. Aran is sponsored by the "Researchers Consolidation Program" from the SNS-Dpt. Salut Generalitat de Catalunya (Exp. CES06/012).

\section{References}

1. Alonso A, Artemis D, Hennerici MG: Molecular imaging of carotid plaque vulnerability. Cerebrovasc Dis 39:5-12, 2015

2. Bae C, Szuchmacher M, Chang JB: Comparative review of the treatment methodologies of carotid stenosis. Int J Angiol 24:215-222, 2015

3. Bennett MR, Sinha S, Owens GK: Vascular smooth muscle cells in atherosclerosis. Circ Res 118:692-702, 2016

4. Brott TG, Halperin JL, Abbara S, Bacharach JM, Barr JD, Bush RL, et al: 2011 ASA/ACCF/AHA/AANN/AANS/ACR/ ASNR/CNS/SAIP/SCAI/SIR/SNIS/SVM/SVS guideline on the management of patients with extracranial carotid and vertebral artery disease: executive summary. Circulation 124:489-532, 2011 (Erratum in Circulation 124:e145, 2011)

5. Choi KH, Park MS, Kim JT, Chang J, Nam TS, Choi SM, et al: Serum triglyceride level is an important predictor of early prognosis in patients with acute ischemic stroke. J Neurol Sci 319:111-116, 2012

6. Christenson SA, Brandsma CA, Campbell JD, Knight DA, 
Pechkovsky DV, Hogg JC, et al: miR-638 regulates gene expression networks associated with emphysematous lung destruction. Genome Med 5:114, 2013

7. Creemers EE, Tijsen AJ, Pinto YM: Circulating microRNAs: novel biomarkers and extracellular communicators in cardiovascular disease? Circ Res 110:483-495, 2012

8. Criqui MH, Ninomiya JK, Wingard DL, Ji M, Fronek A: Progression of peripheral arterial disease predicts cardiovascular disease morbidity and mortality. J Am Coll Cardiol 52:1736-1742, 2008

9. De Guire V, Robitaille R, Tétreault N, Guérin R, Ménard C, Bambace N, et al: Circulating miRNAs as sensitive and specific biomarkers for the diagnosis and monitoring of human diseases: promises and challenges. Clin Biochem 46:846860, 2013

10. De Rosa S, Curcio A, Indolfi C: Emerging role of microRNAs in cardiovascular diseases. Circ J 78:567-575, 2014

11. Di Stefano V, Zaccagnini G, Capogrossi MC, Martelli F: microRNAs as peripheral blood biomarkers of cardiovascular disease. Vascul Pharmacol 55:111-118, 2011

12. Fichtlscherer S, Zeiher AM, Dimmeler S: Circulating microRNAs: biomarkers or mediators of cardiovascular diseases? Arterioscler Thromb Vasc Biol 31:2383-2390, 2011

13. Halliday A, Bulbulia R, Gray W, Naughten A, den Hartog A, Delmestri A, et al: Status update and interim results from the Asymptomatic Carotid Surgery Trial-2 (ACST-2). Eur J Vasc Endovasc Surg 46:510-518, 2013

14. Hergenreider E, Heydt S, Tréguer K, Boettger T, Horrevoets AJ, Zeiher AM, et al: Atheroprotective communication between endothelial cells and smooth muscle cells through miRNAs. Nat Cell Biol 14:249-256, 2012

15. Hermus L, Lefrandt JD, Tio RA, Breek JC, Zeebregts CJ: Carotid plaque formation and serum biomarkers. Atherosclerosis 213:21-29, 2010

16. Jonas S, Izaurralde E: Towards a molecular understanding of microRNA-mediated gene silencing. Nat Rev Genet 16:421433, 2015

17. Kannel WB, Wolf PA, Verter J: Manifestations of coronary disease predisposing to stroke. The Framingham study. JAMA 250:2942-2946, 1983

18. Kawachi I, Colditz GA, Stampfer MJ, Willett WC, Manson JE, Rosner B, et al: Smoking cessation and decreased risk of stroke in women. JAMA 269:232-236, 1993

19. Kubota S, Chiba M, Watanabe M, Sakamoto M, Watanabe N: Secretion of small/microRNAs including miR-638 into extracellular spaces by sphingomyelin phosphodiesterase 3 . Oncol Rep 33:67-73, 2015

20. Li P, Liu Y, Yi B, Wang G, You X, Zhao X, et al: MicroRNA-638 is highly expressed in human vascular smooth muscle cells and inhibits PDGF-BB-induced cell proliferation and migration through targeting orphan nuclear receptor NOR1. Cardiovasc Res 99:185-193, 2013

21. Long G, Wang F, Li H, Yin Z, Sandip C, Lou Y, et al: Circulating miR-30a, miR-126 and let-7b as biomarker for ischemic stroke in humans. BMC Neurol 13:178, 2013

22. Marquardt L, Kuker W, Chandratheva A, Geraghty O, Rothwell PM: Incidence and prognosis of $>$ or $=50 \%$ symptomatic vertebral or basilar artery stenosis: prospective populationbased study. Brain 132:982-988, 2009

23. Marques FZ, Campain AE, Tomaszewski M, Zukowska-Szczechowska E, Yang YH, Charchar FJ, et al: Gene expression profiling reveals renin mRNA overexpression in human hypertensive kidneys and a role for microRNAs. Hypertension 58:1093-1098, 2011

24. Mitchell PS, Parkin RK, Kroh EM, Fritz BR, Wyman SK, Pogosova-Agadjanyan EL, et al: Circulating microRNAs as stable blood-based markers for cancer detection. Proc Natl Acad Sci U S A 105:10513-10518, 2008

25. Moore WS: Issues to be addressed and hopefully resolved in the carotid revascularization endarterectomy versus stenting trial 2. Angiology 67:408-410, 2016

26. O'Brien M, Chandra A: Carotid revascularization: risks and benefits. Vasc Health Risk Manag 10:403-416, 2014

27. Rink C, Khanna S: MicroRNA in ischemic stroke etiology and pathology. Physiol Genomics 43:521-528, 2011

28. Rubin MN, Barrett KM, Brott TG, Meschia JF: Asymptomatic carotid stenosis: what we can learn from the next generation of randomized clinical trials. JRSM Cardiovasc Dis 3:2048004014529419, 2014

29. Sans S, Fitzgerald AP, Royo D, Conroy R, Graham I: [Calibrating the SCORE cardiovascular risk chart for use in Spain.] Rev Esp Cardiol 60:476-485, 2007 (Span)

30. Sarlon-Bartoli G, Bennis Y, Lacroix R, Piercecchi-Marti MD, Bartoli MA, Arnaud L, et al: Plasmatic level of leukocyte-derived microparticles is associated with unstable plaque in asymptomatic patients with high-grade carotid stenosis. J Am Coll Cardiol 62:1436-1441, 2013

31. Sarlon-Bartoli G, Boudes A, Buffat C, Bartoli MA, Piercecchi-Marti MD, Sarlon E, et al: Circulating lipoproteinassociated phospholipase A2 in high-grade carotid stenosis: a new biomarker for predicting unstable plaque. Eur J Vasc Endovasc Surg 43:154-159, 2012

32. Stary HC, Chandler AB, Dinsmore RE, Fuster V, Glagov S, Insull W Jr, et al: A definition of advanced types of atherosclerotic lesions and a histological classification of atherosclerosis. A report from the Committee on Vascular Lesions of the Council on Arteriosclerosis, American Heart Association. Circulation 92:1355-1374, 1995

33. Tsai PC, Liao YC, Wang YS, Lin HF, Lin RT, Juo SH: Serum microRNA-21 and microRNA-221 as potential biomarkers for cerebrovascular disease. J Vasc Res 50:346-354, 2013

34. Wannamethee SG, Shaper AG, Whincup PH, Walker M: Smoking cessation and the risk of stroke in middle-aged men. JAMA 274:155-160, 1995

35. Yang Q, Yin RX, Zhou YJ, Cao XL, Guo T, Chen WX: Association of polymorphisms in the MAFB gene and the risk of coronary artery disease and ischemic stroke: a case-control study. Lipids Health Dis 14:79, 2015

\section{Disclosures}

The authors report no conflict of interest concerning the materials or methods used in this study or the findings specified in this paper.

\section{Author Contributions}

Conception and design: Aran, Krupinski. Acquisition of data: Luque, Farwati. Analysis and interpretation of data: all authors. Drafting the article: Aran, Luque. Critically revising the article: Aran, Krupinski. Reviewed submitted version of manuscript: all authors. Approved the final version of the manuscript on behalf of all authors: Aran. Statistical analysis: Aran, Luque, Farwati. Study supervision: Aran.

\section{Supplemental Information}

\section{Online-Only Content}

Supplemental material is available with the online version of the article.

Supplemental Material. https://thejns.org/doi/suppl/10.3171/ 2018.2.JNS171899.

\section{Correspondence}

Josep M. Aran: Institut d'Investigació Biomèdica de Bellvitge (IDIBELL), Hospital Duran i Reynals, Barcelona, Spain. jaran@ idibell.cat. 